This is the peer reviewed version of the following article: [Australian trampoline injury patterns and trends], which has been published in final form at [10.1111/1753-6405.12404]. This article may be used for noncommercial purposes in accordance with Wiley Terms and Conditions for Self-Archiving. 


\title{
Australian trampoline injury patterns and trends
}

\author{
Karen Ashby, ${ }^{\prime}$ Sophie Pointer, ${ }^{2}$ David Eager, ${ }^{3}$ Lesley Day'
}

\section{T}

rampolining is an enjoyable active recreation and aids in the development of children's co-ordination, balance and motor skills.' The incidence of injury associated with backyard trampolines is reported in surveillance data as a common cause of hospitalisation, primarily among children. ${ }^{2}$ Despite stakeholders' continuing efforts to improve trampoline safety, the trajectory of trampoline injury requiring hospital treatment continues to climb. ${ }^{3}$

In Australia, the response to the growing number of injuries has focused on developing and revising an Australian Standard (AS) for trampolines that includes measurable safety design features engineered to reduce the risk of injury. The first voluntary AS for trampolines, AS 4989-2003: Trampolines, was published in 2003 and set out requirements for components and design as well as specifying information on assembly and maintenance. The revised and improved standard published in October 2006 (AS 49892006: Trampolines - Safety aspects) removed specifications for frame design and focused on safety aspects such as spring and framepadding design, protection of sharp edges, safety marking and labelling, and consumer information. AS 4989-2006 specified that all recreational trampolines offered for sale on the Australian market be supplied with frame padding or a soft-edge system to prevent impact injury. It was recommended that existing trampolines purchased prior to the release of the 2006 Standard be retrofitted with a frame-padding system complying with AS 4989. ${ }^{4}$ The minimum level of consumer safety information was also detailed and included safety warnings on the trampoline packaging, instruction on installation, maintenance information for ongoing safe use, including the need for active adult

\begin{abstract}
Objectives: To examine national trampoline injury patterns and trends in the context of improved product safety standards and trampoline design modifications.

Method: Review of National Hospital Morbidity data.

Results: There were an average 1,737 trampoline injuries reported nationally each year from 2002 to 2011. Both injury frequency and rate grew. Statistically significant rate increases were observed among all age groups, although both are highest among children aged 5-9 years. From 2008/09 there is a possible decreasing trend among the $5-9$ age group. Falls predominate and $81 \%$ of falls result in fracture. Non-fall injuries increased annually as a proportion of all hospitalised injury although they did not comprise more than $2.4 \%$ in any one year.

Conclusions: History provides no evidence of an observable effect of voluntary Australian Standards for trampoline safety on population rates for trampoline injury. The major design modification - netted enclosures - could contribute to the risk of injury by leading parents to falsely believe that a netted enclosure eradicates the risk of injury.

Key words: trampoline injury, Australian Standards, child injury hospitalisation
\end{abstract}

supervision. Fur ther minor amendments were made to AS 4989-2006 in 2008 and 2010.

It was anticipated that revisions to the AS would result in reductions in trampolinerelated injury, although the impact was not expected to be seen for several years, given the lifespan of existing trampolines. Contemporaneously, trampolines with safety enclosure 'netting' to minimise the risk of children falling off - one of the most common mechanisms of injury - were introduced on to the Australian market. Despite these changes, expected reductions in trampoline-related injuries have not been seen.

In response to the lack of improvement in safety, the Standards Australia Trampoline Committee CS-100 has recently revised AS 4989 with the view that it will be adopted as a Mandatory Standard by the Australian Competition and Consumer Commission (ACCC). The revised Standard includes the requirement of safety enclosures that meet minimum specifications, UV degradation testing of all plastic components, structural integrity testing, an easier frame-padding test and an enclosure test. It is anticipated that this will be a two-stage process: first, publication of the Standard, then the adoption of a regulatory response that will mandate some or all of the Standard's specifications.

Hence, it is timely to examine national trampoline injury patterns and trends for serious (hospital-admitted) trampoline injury to provide a benchmark prior to the imminent introduction of the mandatory standard.

\section{Method}

Data were extracted from the National Hospital Morbidity Database (NHMD) for the time period 1 July 2002 to 30 June 2011. The NHMD is compiled from data supplied by the state and territory health authorities. It is a collection of electronic confidentialised summary records for separations (i.e. episodes of care as a result of an admission) in public

1. Monash injury Researd Institute, Monash University, Victoria

2. Research Centre for Injury Studies, Finders University, South Australia

3. Facuity of Engineering and IT, University of Technolo $\delta y$, Sydney, New South Wales

Correspondence to: Professor Lesley Day, Victorian IJ ury Surveillance and Falls Prevention Research Units, Monash Injury Research Institute, Building 70, Monash University,

Wellington Road, Clayton. VIC 3800; e-mail Hestey.dayemonash.edu

Submitted: October 2014; Revision requested: lanuary 2015; Accepted: February 2015

The authors have stated they have no conflict of interest. 
and private hospitals in Australia. People who are evaluated in hospital emergency departments and then discharged are not included in the NHMD. Cases of trampolinerelated injury were defined as those separated from hospital after an admission to hospital with an International Classification of Diseases (ICD) version 10 (Australian Modification) injury diagnosis (principal diagnosis code in the range S00-T75 or T79), AND with an 'External Injury Cause' code of Wo9.6 Fall involving trampoline anywhere in the record OR with an 'Activity' code of U57.06 Trampoline and mini-trampoline from the Acrobatic, sports, gymnastics coding block anywhere in the record. ICD version 10 (Australian Modification) has been used in Australia since 1998/99 and these trampolinerelated codes have been in use since 2002 . There were no changes to data acquisition policy for the NHMD over the study period.

Multiple episodes of hospital care for the same injury event ('case'), as the result of inter-hospital transfers, readmissions or similar cannot be identified in the NHMD. Case selection criteria was adjusted to some extent for potential multiple counting by omitting records with a mode of admission of 'transfer from another acute hospital'.

Cases were assigned to either a fall or non-fall category. Fall cases had an external injury cause code of W09.6 OR an activity code of U57.06 AND an external cause code in the range W17-W19. Non-fall cases had an activity code of U57.06 AND an external cause of injury code other than W09.6 or W17-W19. A small number of cases $(n=14)$ were identified within the non-fall category with an external cause code of W03 Fall on same level due to a collision with another person. As the collision was the primary event in the chain of events leading to injury they were retained in the non-fall category.

The denominators used for calculating rates were mid-year age and sex-specific population estimates for Australia sourced from the Australian Bureau of Statistics (ABS) derived from census figures and inter-census quarterly surveys.

Descriptive analysis was undertaken using the Statistical Package for Social Sciences (SPSS) version 20. Trends were determined using a log-linear regression model of the rate data assuming a Poisson distribution of injuries. The statistics related to the trend curves, slope and intercept, estimated annual percentage change and the $p$-value were calculated using the regression model in SAS 9.2. A trend was considered to be statistically significant if the $p$-value of the slope of the regression model was less than 0.05 .

\section{Results}

There were 15,636 hospitalised trampoline injuries in Australia over the nine-year period with an annual average of 1,737 cases. Figure 1 shows the breakdown by financial year and indicates an increase from a low of 1,392 cases in 2002/03 to 1,982 cases in 2010/11. The highest number of cases $(n=2,098)$ was recorded in 2008/09.

Falls predominated as the mechanism of injury. Non-falls increased as a proportion of all hospitalised injury, although they did not comprise more than $2.4 \%$ in any one year. As shown in Table 1 and Figure 2, both the frequency and rate is highest in the 5-9 age group, representing almost half all trampoline injury admissions. Among non-fall injuries children aged 10-14 years represented almost one-third of cases. Persons aged 15+ years comprised a higher proportion of nonfall, than fall injury. This pattern was observed for both genders (Figure 3). Males were overrepresented in both categories particularly for non-fall injury (Table 1).

Fall injury mostly resulted in fracture $(81 \%$ of fall injuries), predominantly to the upper limb (70\% of all fall injuries) (Table 1 ).

Non-fall injuries were mostly collisions with another trampoline user and overexertions (Table 1). Like fall injury, fractures predominated among non-fall injuries (52\%); unlike fall injury, $40 \%$ of injuries were to the lower limb. Dislocation, sprain or strains were more common in the non-fall group. Head injury represented $11 \%$ of injury in each category.

\section{Trend analysis}

Injury rates increased for all age groups over the time period examined and the trends reached statistical significance for each age group (Figure 3, Table 2). Table 2 indicates annual percentage increases ranged from $2.4 \%$ in the 5-9 age group to $9.0 \%$ in the 15-19 age group. The highest percentage change over the period was for 15-19 year olds, followed by $0-4$ year olds. Among the 5-9 year age group we observed a possible decreasing trend emerging since 2008/09; however, more years of observation are required for confirmation.

\section{Discussion}

Despite a published Australian Standard specifying minimum safety and product labelling requirements, both the frequency and population rate of injury from

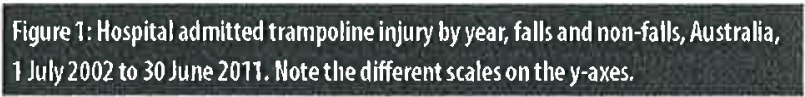

\section{Figure 2: Trends in trampoline injury hospital admission rates per 100,000 population, Australia, July 1,2002 to Jume 30, 2011.}

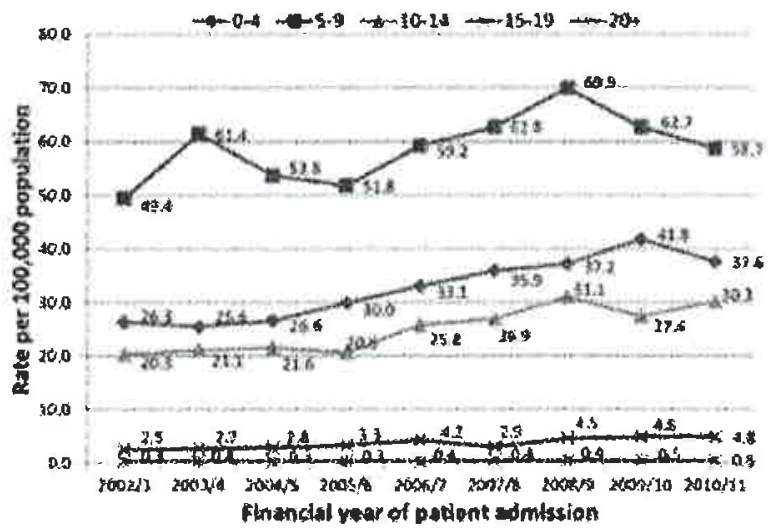


trampolines grew over the study period. Statistically significant increases in admission rates were observed among all age groups. There is a potentially promising decrease among the predominate group: 5-9 year olds.

Similar trends have been observed in other developed nations. Chalmers ${ }^{5}$ reported a three-fold increase in the admission rate in New Zealand in the decade to 1998, Smith ${ }^{6}(1998)$ noted a $98 \%$ increase in child trampoline injury presenting to Emergency Departments (EDs) in the United States in the period 1990-1995 and, similarly, Leonard and Joffe ${ }^{7}$ reported a 374\% increase in child presentations to EDs for trampoline injury in Canada over the period 1990 to 1998.

Prevention efforts are potentially showing some evidence of injury reduction in the predominate age group (5-9 year olds); however, the increases in serious injury overall and among the other age groups suggest otherwise. Wootton and Harris ${ }^{8}$ warned that the presence of netted enclosures could be increasing the risk of injury by leading parents to believe that injuries only occur when children fall from, or make contact with the frame. Wootton and Harris suggested parents may falsely believe that the netted enclosure eradicates the risk of injury and lessens the need for constant supervision and adherence to the recommended operating guidelines. This unintended effect may be contributing to the increases in the number of injuries among the youngest children. A recent study by Klimek ${ }^{9}$ provides support for these hypotheses. Data collected by questionnaire from parents of children (age $<16$ years) who had presented to hospital between 2003 and 2009 with backyard trampoline injuries was correlated with radiographic findings. Parental supervision was evident in only $27 \%$ of incidents. In addition, $75 \%$ of the incidents and more than $90 \%$ of the fractures occurred while more than one child was on the trampoline.

An analysis of ED data in Victoria showed an annual increase of $18 \%$ in multi-user injuries over a nine-year period, which was significantly higher than the overall annual increase of $13 \% .{ }^{3}$ This analysis also showed that the youngest age group ( $0-4$ year olds) were over-represented for this type of injury compared to other types of trampoline injury. Similarly, there may be an increased likelihood that older children and teenagers attempt risky manoeuvres if the fear of a fall from the trampoline is ameliorated.
More than half the non-fall injuries were fractures, but injuries were more likely to be to the lower limb compared with fall injuries. This may be consistent with the injured person being 'double bounced', where one user descends, hits the mat feet first and is immediately catapulted up even higher by the force of another person landing. The smaller, lightest person is most at risk from this practice where considerable energy is transferred.

Falls remain the leading cause of trampolinerelated injury in Australia. Analysis of Victorian ED presentations that went on to be hospitalised indicated that $71 \%$ of admitted fall cases were due to a fall from a trampoline, while $18 \%$ were the result of falls on the trampoline surface. ${ }^{3}$ This pattern is similar to that reported in New Zealand; 5 however, this contrasts to reports from the northern hemisphere (US, Canada and United Kingdom) where injuries occurring on the body of the trampoline predominate..$^{8,10-12}$ This may be attributable to the earlier recommendations for trampoline safety enclosures that have been part of the American Society for Testing and Materials (ASTM) Trampoline Standard since 2003.

There is an unavoidable time lag with introduction of technical interventions such as containment enclosure design, UV inhibitor protection, structural integrity and stability to over-turning. Australian households that own a trampoline generally retain this unit for many years. This system inertia creates a time lag to any safety intervention. For example, if there are 800,000 non-conforming trampolines in backyards across Australia and we add 160,000 complying trampolines and withdraw 80,000 trampolines per year it will take many years to observe the true impact of the interventions, and the injury data must be considered in this context.

\begin{tabular}{|c|c|c|c|c|c|c|}
\hline & \multicolumn{2}{|c|}{ Falls $(n=15,333)$} & \multicolumn{2}{|c|}{ Non-falls $(n=303$ ) } & \multicolumn{2}{|c|}{ Total $(n=15,636)$} \\
\hline & N & क & N & 96. & H. & 8. \\
\hline \multicolumn{7}{|l|}{ Age group } \\
\hline $0-4$ & 4,015 & 26.2 & 49 & 16.2 & 4,064 & 26.0 \\
\hline $5-9$ & 7,142 & 46.6 & 77 & 25.4 & 7,279 & 46.2 \\
\hline $10-14$ & 3,154 & 20.6 & 96 & 31.7 & 3,250 & 20.8 \\
\hline $15-19$ & 473 & 3.1 & 42 & 13.9 & 515 & 3.3 \\
\hline $20+$ & 549 & 3.5 & 39 & 12.9 & 588 & 3.8 \\
\hline Mean age & & 8.0 & & 12.5 & & 8.1 \\
\hline Medlan & & 7 & & 11 & & 7 \\
\hline \multicolumn{7}{|l|}{ Gender } \\
\hline Male & 8,593 & 56.0 & 202 & 66.7 & 8,795 & 56.2 \\
\hline Female & 6,739 & 44.0 & 101 & 33.3 & 6,840 & 43.8 \\
\hline Unspedfied & 1 & 0 & 0 & 0 & 1 & 0 \\
\hline \multicolumn{7}{|l|}{ Cause } \\
\hline Fall involving trampoline & 15,333 & 100.00 & - & - & 15,333 & 98.1 \\
\hline Struck by/collision wth person & - & - & 93 & 30.7 & 93 & 0.6 \\
\hline Strudk by/cush In/collislon with object & - & - & 62 & 20.5 & 62 & 0.4 \\
\hline Over-exertion & - & - & 62 & 20.5 & 62 & 0.4 \\
\hline 0 ther and unspedfied & . & - & 86 & 28.4 & 86 & 0.5 \\
\hline \multicolumn{7}{|l|}{ Nature of main injury } \\
\hline Fracture & 12,424 & 81.0 & 158 & 52.1 & 12,582 & 80.5 \\
\hline Open wound & 658 & 4.3 & 29 & 9.6 & 687 & 4.4 \\
\hline Dislocatton, sprain/straln & 500 & 3.3 & 45 & 14.8 & 545 & 3.5 \\
\hline Intracanlal & 379 & 2.5 & 9 & 3.0 & 388 & 2.5 \\
\hline Superficlal Injury & 217 & 1.4 & 9 & 3.0 & 226 & 1.4 \\
\hline Other and unspedfied & 1,155 & 7.5 & 53 & 17.5 & 1,208 & 7.7 \\
\hline \multicolumn{7}{|l|}{ Body region injured } \\
\hline Head/face & 1,676 & 10.9 & 76 & 25.1 & 1,752 & 11.2 \\
\hline Trunk ind. neck & 867 & 5.6 & 49 & 16.2 & 916 & 5.9 \\
\hline Upper limb & 10,757 & 70.1 & 57 & 18.8 & 10,814 & 69.2 \\
\hline Lower limb & 2,003 & 13.1 & 119 & 39.3 & 2,122 & 13.6 \\
\hline Other and unspedfied & 30 & 0.2 & 2 & 0.6 & 32 & 0.2 \\
\hline
\end{tabular}


Table 2: Percentage change and annual percentage increase in hospital admitted trampoline injury rates by age group, Australia, 1 July 2002 to 30 June 2011. Perceitagecdange Annual percentige over periad $(95 \%$ a) incease

\begin{tabular}{lrr}
\hline $0-4$ years & $74.7(50.5,95.8)$ & $6.4 \%$ \\
\hline $5-9$ years & $24.0(1.2,50.5)$ & $24 \%$ \\
\hline $10-14$ years & $66.1(39.9,91.4)$ & $5.8 \%$ \\
\hline $15-19$ years & $117.2(60.5,173.8)$ & $9.0 \%$ \\
\hline $20+$ years & $72.1(30.5,118.3)$ & $6.2 \%$ \\
\hline
\end{tabular}

\section{Limitations}

The ICD-10AM coding system is limited in its ability to identify trampoline-related injury in several ways. Firstly, the trampolinerelated fall code relates to any fall involving a trampoline and there is no resolution within the data as to what that actually covers (e.g. could be a fall from, fall on, or fall after a collision with another person). In fact, in the case of fall after collision, coders may deem it preferential to code to a fall as there is no 'collision on trampoline' code but there is a specific'fall involving trampoline' code.

The introduction of a trampoline activity code provided a way to capture non-fall trampoline cases. However activity coding is notoriously poorly completed compared to external cause coding. An investigation of NSW data indicated that for all children and young people during $2009-10,52 \%$ of all-cause injury cases had an unspecified activity at the time of injury. ${ }^{13}$ Due to the poor coding of activity and lack of specificity in external cause coding, a selection bias may have been present in the study.

The activity code used in this study to identify all cases of trampoline-related injuries includes mini-trampolines as well as the more common large trampolines. Mini-trampolines are not covered by the Standard AS 4989. They cannot be readily excluded; however, estimates based on Victorian emergency department data indicate that minitrampolines are implicated in only $0.06 \%$ of admitted cases, and hence would account for about 10 cases in the present analysis.

Australia also lacks exposure data, making it impossible to determine what proportion of the increase in frequency of injury is related to increased exposure alone. The population rates presented in our study do not account for changes in the numbers of people using trampolines or the amount of time spent using trampolines. Further, data on both the number of conforming and non-conforming

Figure 3: Trampoline injury hospital admissions by fall and non-fall, age group and gender, Australia, July 1, 2002 to June 30,2011

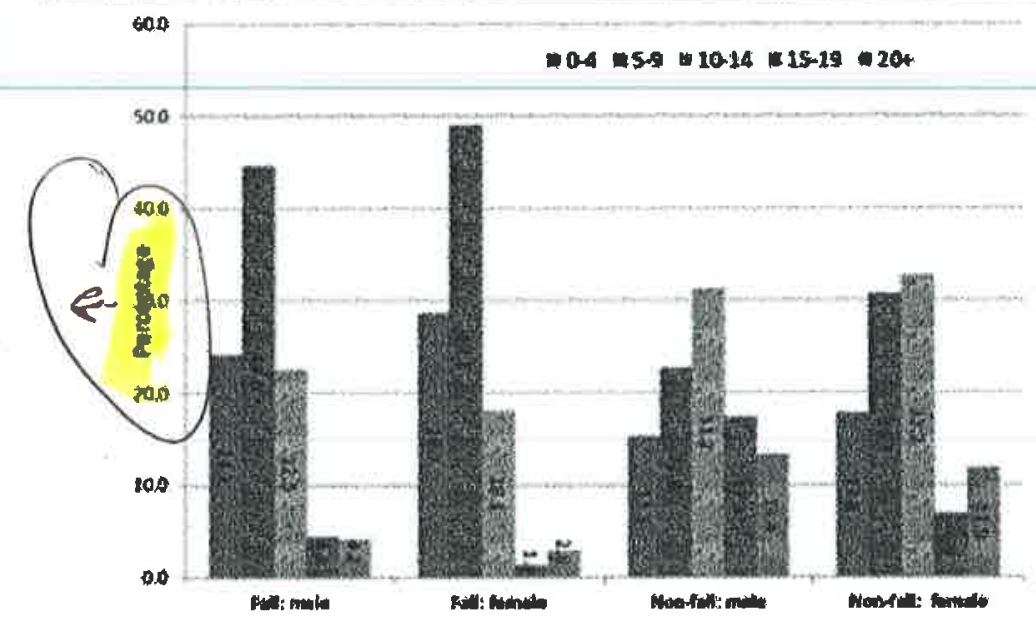

trampoline stock in Australian backyards is not available.

\section{Conclusion and implications}

History provides no evidence of an observable effect by the voluntary Australian Standard for trampoline safety on population rates for trampoline-related injuries in Australia over the past decade. Injury trends may begin to level out and, over time, decrease with the mandating of the new Standard by the ACCC in 2014. The nature of trampoline injury may also change. This study provides a benchmarking opportunity for examining change.

Trampoline-related injuries can, and will continue to, occur on products that fully comply with the mandated Standard. For example, the introduction of safety enclosures - a design feature to reduce injuries - may have had the unintended consequence of increasing risk to younger users. It is thus important that the product mandating be coupled or linked to other injury prevention strategies such as public awareness and education. In addition, the buy-back and destruction of old and dangerous products would accelerate the reduction in injuries. Timely product recalls on any trampolines that are found to not comply with the Standard would encourage and reward manufacturers who do comply, while penalising those who do not.

\section{Acknowledgements}

The authors thank Angela Clapperton from the Monash Injury Research Institute at
Monash University for assistance with trend analysis, and acknowledge the Australian Institute of Health and Welfare (AlHW) for data provision. L. Day was supported by the Victorian Injury Surveillance Unit's core grant from the Victorian Department of Health.

\section{References}

1. Eager D, Chapman C, Bondoc K. Characterisation of trampoline bounce using acceleration. In: Proceeding of 7 th Australasion Congresson AppliedMechanics 2012 Dec 9-12; Adelaide, Australia.

2. Pointer S, Helps Y. Trends in Hospitalised Childhood Injury in Australia 1999-07. Injury Researd ond Statistics Series No: 75. Catalogue No: INUCAT 151. Canberra (AUST):Australian Institute of Health and Welfare; 2012

3. Ashby K, Day L Trampoline Injury [Internet). Hazard No: 13. Melboume (AUST): Monash Injury Research Institute; 2013 [cited 2014 Jan]. Available from: httpi/l wwwmenashedu.au/miri/research/research-areas/ home-spert-and teisure-safety/visu/hazardBaz75,pdf

4. Eager D. Trampoline StandardAS 4989-2006. Australas Parks Leisure. 2007;Autumn:7-8.

5. Chalmers DJ, Hume PA, Wilson BD. Trampolines in New Zealand: A decade of injufies-Ars Sports Med. 1994:28:234-8.

6. Smith GA. Injuries to Children in the United States Related to Trampolines, 1990-1995: A National Epidemic Pediatrico. 1998;101:406-12.

7. Leonard $\mathrm{H}_{4}$ JoffeAR. Children presenting to a Canadian hospital with trampoline-relatedcervical spine injuries JPaediatt Child Health. 2009;14:84-8.

8. Wootton $M$, Harris D. Trampolining injuries presenting to a children's emergency department. Emerg Med J. 2009;26:728-31.

9. Klimek PM, Juen D, Stranzinger $E$, Wolf $R$, et al. Trampoline related injuries in children:Riskfactors and radiographic findings. World JPediatr. 2013;9:169-74.

10. Fumival RA, Street KA, SchunkJE Too Many Paediatric Trampoline Injuries. Pediatrics. 1999;103:5.

11. Alexander K, Eager D, Scarrott C. Sushinsky G. Effectiveness of pads and enclosures as safety interventions on consumer trampolines. Inj Prev. 2010;16:185-9.

12. Bogacz A. Trampoline injuries: How to avoid injury [letter].BM. 2009;338:b2197.

13. Hanis CE, Pointer SC. Serious childhood community injury in New South Wales 2009-10. Injury Research and Statistics.Series Na:76. Catalogue No: INUCAT 152 Canberra (AUST): Australian Institute of Health and Welfare; 2012 